\title{
Irrationality measures of the values of hypergeometric functions
}

\author{
by \\ Masayoshi Hata (Kyoto)
}

1. Introduction. Recently the author [4] studied the rational approximations to the values of logarithm, dilogarithm and trilogarithm at particular rational points, such as $\log 2, \pi / \sqrt{3}, \zeta(2)$ and $\zeta(3)$, using the following Legendre type polynomials:

$$
\begin{aligned}
H_{n, m}(x) & =\frac{1}{n !}\left(x^{n-m}(1-x)^{n+m}\right)^{(n)} \\
& =\sum_{j=0}^{n}(-1)^{m+j}\left(\begin{array}{c}
n+m \\
m+j
\end{array}\right)\left(\begin{array}{c}
n+j \\
n
\end{array}\right) x^{j} .
\end{aligned}
$$

In the present paper we continue the similar researches by introducing another kind of Legendre type polynomial:

$$
\begin{aligned}
P_{n, m, \delta}(x) & =\frac{x^{\delta}}{n !}\left(x^{n-\delta}(1-x)^{n+m}\right)^{(n)} \\
& =\sum_{j=0}^{n+m}(-1)^{j}\left(\begin{array}{c}
n+m \\
j
\end{array}\right)\left(\begin{array}{c}
n+j-\delta \\
n
\end{array}\right) x^{j},
\end{aligned}
$$

where $\delta=a / b \in(0,1), a, b \in \mathbb{N}$, is a fixed rational number. Applying the above polynomials to some one-dimensional integrals we can construct rational approximations to the values of the following particular Gaussian hypergeometric function:

$$
{ }_{2} F_{1}(1,1-\delta, 2-\delta ; x)=(1-\delta) \sum_{n=1}^{\infty} \frac{x^{n-1}}{n-\delta}
$$

at rational points $x$. Here we restrict ourselves to the case $x=1 / s$, where $s$ is an integer satisfying

$$
s \equiv 1\left(\bmod b \cdot \prod_{\substack{p \mid b \\ p \text { prime }}} p\right) .
$$


Although this restriction is stronger than the condition (b) of Huttner's theorem ([5], p. 169), this enables us to obtain comparatively good irrationality measures of the corresponding values (1.3); indeed, some of them improve the earlier results obtained by Huttner [5] and by the author [3].

Note that the polynomials (1.1) and (1.2) for $\delta=1 / 2$ are closely related. In fact, since

$$
\begin{aligned}
\int_{0}^{1} x^{j} P_{n, m, 1 / 2}((2 x & \left.-1)^{2}\right) d x \\
= & \frac{1}{4} \int_{0}^{1}\left\{\left(\frac{1+\sqrt{y}}{2}\right)^{j}+\left(\frac{1-\sqrt{y}}{2}\right)^{j}\right\} P_{n, m, 1 / 2}(y) \frac{d y}{\sqrt{y}}
\end{aligned}
$$

and since the function in \{\} is a polynomial of $y$ of degree $[j / 2]$, it follows that $P_{n, m, 1 / 2}\left((2 x-1)^{2}\right)$ is orthogonal to $x^{j}$ for $0 \leq j<2 n$. Moreover, it vanishes at $x=0$ and $x=1$ with order at least $m$. Therefore it follows from Lemma 2.1 in [4] that $P_{n, m, 1 / 2}\left((2 x-1)^{2}\right)$ and $x^{m} H_{2 n+m, m}(x)$ differ only by a constant multiple. Comparing their coefficients of the highest order, we thus have

$$
P_{n, m, 1 / 2}\left((2 x-1)^{2}\right)=(-1)^{n+m} \frac{\left(\begin{array}{c}
n+m \\
m
\end{array}\right)}{\left(\begin{array}{c}
2 n+2 m \\
m
\end{array}\right)}(4 x)^{m} H_{2 n+m, m}(x) .
$$

Concerning the values of logarithm at rational points such as $\log 2$ and $\pi / \sqrt{3}$, our polynomial $P_{n, m, 1 / 2}(x)$ reproduces the same irrationality measures as obtained in the previous paper [4]. For the approximations by algebraic numbers to such numbers, see Reyssat [6].

Our polynomial $P_{n, m, 1 / 2}(x)$ can also produce good irrationality measures of some numbers involving logarithm at algebraic points. For example, as a special case of our main theorem, we have

TheOREM 1.1. For any $\varepsilon>0$, there exists a positive integer $q_{0}(\varepsilon)$ such that

$$
\left|\sqrt{5} \log \left(\frac{1+\sqrt{5}}{2}\right)-\frac{p}{q}\right| \geq q^{-\nu-\varepsilon}
$$

for any integer $q \geq q_{0}(\varepsilon)$ and for all $p \in \mathbb{Z}$, where $\nu$ is given by

$$
\nu=1+\frac{\log \alpha_{0}+\beta_{0}}{\log \alpha_{0}-\beta_{0}}
$$

with $\alpha_{0}=(65821+2929 \sqrt{505}) / 108$ and $\beta_{0}=6-\pi(2 / \sqrt{3}-1 / 2)$. (Numerically one has $\nu=4.4937113 \ldots)$ 
Similarly our polynomials $P_{n, m, \delta}(x)$ in the cases $b=3$ and $b=4$ can produce the following new irrationality measures of some numbers involving $\pi$.

TheOREM 1.2. For any $\varepsilon>0$, there exists a positive integer $q_{1}(\varepsilon)$ such that

$$
\left|\left(\frac{\pi}{\sqrt{3}} \pm \log 3\right)-\frac{p}{q}\right| \geq q^{-\mu-\varepsilon}
$$

for any integer $q \geq q_{1}(\varepsilon)$ and for all $p \in \mathbb{Z}$, where $\mu$ is given by

$$
\mu=1+\frac{\log \left(\sqrt{3} \alpha_{1}\right)+\beta_{1}}{\log \alpha_{1}-\beta_{1}}
$$

with $\alpha_{1}=3^{-9 / 4}(960991+129580 \sqrt{55})$ and $\beta_{1}=9-\pi(3 / 2+\sqrt{3} / 6-$ $(\cot (\pi / 9)+\cot (2 \pi / 9)+\cot (4 \pi / 9)) / 4) . \quad$ (Numerically one has $\mu=$ $4.5586217 \ldots)$

TheOREM 1.3. For any $\varepsilon>0$, there exists a positive integer $q_{2}(\varepsilon)$ such that

$$
\left|\left(\frac{\pi}{\sqrt{3}} \pm \sqrt{3} \log (2+\sqrt{3})\right)-\frac{p}{q}\right| \geq q^{-\xi-\varepsilon}
$$

for any integer $q \geq q_{2}(\varepsilon)$ and for all $p \in \mathbb{Z}$, where $\xi$ is given by

$$
\xi=1+\frac{\log \alpha_{2}+\beta_{2}}{\log \alpha_{2}-\beta_{2}}
$$

with $\alpha_{2}=2^{-21 / 2}(153333125+7734633 \sqrt{393})$ and $\beta_{2}=32 / 3-\pi(1 / \sqrt{2}+$ $\sqrt{1+1 / \sqrt{2}}-5 / 4)$. (Numerically one has $\xi=6.1382145 \ldots)$

Unfortunately, our method seems to be inappropriate to obtain any irrationality measures of other kind of numbers such as $\log 3$ and $\sqrt{3} \log (2+\sqrt{3})$. For the rational approximations to such numbers, see Rhin [7].

In applications of the polynomials (1.2) to some one-dimensional integrals the exact asymptotic behaviour of the remainder terms of our rational approximations can be easily obtained by using the following fact:

$$
\lim _{n \rightarrow \infty}\left(\int_{0}^{1}|f(t)|^{n} \varphi(t) d t\right)^{1 / n}=\max _{0 \leq t \leq 1}|f(t)|
$$

where $f(t)$ is continuous and $\varphi(t)$ is a non-negative integrable weight function such that there exists a point $t_{0} \in[0,1]$ at which $|f(t)|$ attains its maximum and $\varphi(t) \not \equiv 0$ in any neighbourhood of $t_{0}$. So we do not need any further information on the polynomials (1.2), such as the uniqueness, the distribution of zeros, the recurrence formula, etc., except for some arithmetical properties of their coefficients. (1.5) will be easily proved by modifying the proof of the usual case in which $\varphi(t) \equiv 1$. 
2. Arithmetical properties of the coefficients. In this section we investigate some arithmetical properties of the coefficients of $P_{n, m, \delta}(x)$ where $\delta=a / b \in(0,1)$ and $a, b$ are positive integers with $(a, b)=1$. First of all, we need the following elementary arithmetical lemma, which gives a generalization of the well-known fact that the exponent of any prime $p$ in the resolution of $n$ ! into its prime factors is precisely equal to $v(n, p) \equiv$ $[n / p]+\left[n / p^{2}\right]+\ldots$

Lemma 2.1. Let $a<b$ be positive integers with $(a, b)=1$. Then

$$
\prod_{k=1}^{n}(k b-a)=\prod_{\substack{p \nmid b \\ p \text { prime }}} p^{v(n, p ; a, b)}
$$

for every $n \geq 1$, where

$$
v(n, p ; a, b)=\sum_{j=1}^{\infty}\left[\frac{n}{p^{j}}+\frac{w_{j}}{b}\right]
$$

and $w_{j} \equiv w_{j}(p ; a, b) \in[1, b)$ is the unique solution of the congruence

$$
p^{j} w_{j} \equiv a(\bmod b) .
$$

Proof. The proof is easily given by modifying the method of the resolution of $n$ ! into its prime factors.

Since $(k b-a, b)=1$ for every $k \geq 1$, no prime factors of $b$ appear in the resolution of the number (2.1) into its prime factors. So it is sufficient to show that the following $n$ integers:

$$
b-a, 2 b-a, \ldots, n b-a
$$

include just $\left[n / p^{j}+w_{j} / b\right]$ multiples of $p^{j}$ for each $j \geq 1$ and for any prime number $p$ with $p \nmid b$. To see this, let $u_{k}$ be the least non-negative residue of $k b-a$ to modulus $p^{j}$ for $k \geq 1$. Then, since $(p, b)=1$, the $p^{j}$ integers $u_{1}, u_{2}, \ldots, u_{p^{j}}$ form a complete system $\left(\bmod p^{j}\right)$; so there exists a unique $k_{j} \in\left[1, p^{j}\right]$ such that $u_{k_{j}}=0$. Therefore the integers (2.2) include just $N$ multiples of $p^{j}$, where $N$ is the largest integer satisfying $k_{j}+(N-1) p^{j} \leq n$; hence $N=\left[\left(n-k_{j}\right) / p^{j}\right]+1$. Thus

$$
\frac{n-k_{j}}{p^{j}}+1 \leq N+\frac{p^{j}-1}{p^{j}} .
$$

On the other hand, put $p^{j} w_{j}=a+b r_{j}$ for some integer $r_{j}<p^{j}$. Then $b\left(p^{j}-r_{j}\right)-a=p^{j}\left(b-w_{j}\right)$; hence $k_{j}=p^{j}-r_{j}$. Thus we have

$$
N \leq \frac{n-k_{j}}{p^{j}}+1 \leq \frac{n-k_{j}}{p^{j}}+1+\frac{a}{b p^{j}}<N+\frac{p^{j}-1}{p^{j}}+\frac{1}{p^{j}}=N+1,
$$


which then implies

$$
N=\left[\frac{n-k_{j}}{p^{j}}+1+\frac{a}{b p^{j}}\right]=\left[\frac{n}{p^{j}}+\frac{w_{j}}{b}\right]
$$

as required.

In the above lemma we note that the sequence $\left\{w_{j}\right\}$ also satisfies the congruence $p w_{j+1} \equiv w_{j}(\bmod b)$ with $w_{0}=a$ and that $w_{j}$ runs through some subset of a complete set of residues prime to $b$ periodically. The period of $\left\{w_{j}\right\}$ is equal to the order of $p(\bmod b)$; that is, the smallest positive integer $r$ for which $p^{r} \equiv 1(\bmod b)$.

Lemma 2.2. Let $\lambda>1$ be a real parameter. Then the following $n+1$ positive integers:

$$
\left(\begin{array}{c}
n+[n / \lambda] \\
{[n / \lambda]+j}
\end{array}\right) \frac{1}{j !} \prod_{\substack{p \mid b \\
p \text { prime }}} p^{v(j, p)} \prod_{k=n-j+1}^{n}(k b-a),
$$

$0 \leq j \leq n$, have a common divisor $d_{n}(\lambda ; a, b)$ such that

$$
\lim _{n \rightarrow \infty} \frac{1}{n} \log d_{n}(\lambda ; a, b)=\frac{1}{\lambda \psi(b)} \sum_{\substack{1 \leq k<b \\(k, b)=1}} \int_{E(\lambda, k / b)} \frac{d y}{y^{2}}
$$

where $\psi(b)$ is Euler's function and $E(\lambda, k / b)$ is a countable union of open intervals defined by

$$
E(\lambda, k / b)=\{x>0 ;\{\lambda x\}>k / b \text { and } 1<\{x\}+\{\lambda x\}<1+k / b\}
$$

$(\{x\}$ denotes the fractional part of $x)$.

Proof. Clearly the numbers (2.3) are positive integers, since

$$
v(n, p ; a, b) \geq v(n-j, p ; a, b)+v(j, p)
$$

for any prime number $p$ with $p \nmid b$. For any integer $k \in[1, b)$ with $(k, b)=1$, we first define

$$
\begin{aligned}
E_{n}(\lambda, k / b)=\{x>0 ;\{\lambda x\} & >k / b \text { and } \\
1 & +1 / \sqrt{n}<\{x\}+\{\lambda x\}<1+k / b-1 / \sqrt{n}\}
\end{aligned}
$$

for every $n>4 b^{2}$. Then $E_{n}(\lambda, k / b)$ is a countable union of disjoint open intervals and $\left\{E_{n}(\lambda, k / b)\right\}$ is a monotone increasing sequence of open sets with respect to $n$ satisfying

$$
\bigcup_{n} E_{n}(\lambda, k / b)=E(\lambda, k / b) .
$$

Let $S_{n}(\lambda, k ; a, b)$ be the set of all prime numbers $p$ such that $p>\sqrt{b n}$, $p \equiv k^{\prime}(\bmod b)$, and that $n / \lambda p \in E_{n}(\lambda, k / b)$, where $k^{\prime} \in[1, b)$ is the unique solution of the congruence $-k k^{\prime} \equiv a(\bmod b)$. Note that if $k$ runs through a 
complete set of residues prime to $b$, then $k^{\prime}$ also runs through the same set. We then define

$$
d_{n}(\lambda ; a, b)=\prod_{\substack{1 \leq k<b \\(k, b)=1}} \prod_{p \in S_{n}(\lambda, k ; a, b)} p .
$$

We first show that each prime number $p \in S_{n}(\lambda, k ; a, b)$ divides all the integers (2.3) for $0 \leq j \leq n$. To see this, for an arbitrarily fixed $j \in[0, n]$, let $\omega, \eta$ and $\theta$ be the fractional parts of $n / p,[n / \lambda] / p$ and $j / p$ respectively. Then

$\left\{\frac{n}{\lambda p}\right\}>1+\frac{1}{\sqrt{n}}-\omega>\frac{1}{\sqrt{n}} \quad$ and $\quad\left\{\frac{n}{\lambda p}\right\}<1+\frac{k}{b}-\frac{1}{\sqrt{n}}-\omega<1-\frac{1}{\sqrt{n}}$, since $n / \lambda p \in E_{n}(\lambda, k / b)$. Therefore, since

$$
\left|\frac{n}{\lambda p}-\frac{[n / \lambda]}{p}\right|=\frac{\{n / \lambda\}}{p}<\frac{1}{p}<\frac{1}{\sqrt{n}},
$$

we have $|\{n / \lambda p\}-\eta|<1 / \sqrt{n}$; hence

$$
\omega+\eta>\left\{\frac{n}{p}\right\}+\left\{\frac{n}{\lambda p}\right\}-\frac{1}{\sqrt{n}}>1
$$

and

$$
\omega+\eta-\frac{k}{b}<\left\{\frac{n}{p}\right\}+\left\{\frac{n}{\lambda p}\right\}+\frac{1}{\sqrt{n}}-\frac{k}{b}<1 .
$$

Thus we obtain

$$
[\omega+\eta]-[\eta+\theta]-[\omega-\theta]+[\omega-k / b]-[\omega-\theta-k / b] \geq 1,
$$

since $[\omega-\theta] \leq 0$ and $[\omega-k / b]=0=[\omega+\eta-k / b] \geq[\eta+\theta]+[\omega-\theta-k / b]$. Hence, since $w_{1}(p ; a, b)=b-k$ and $v(n, p ; a, b)=[n / p-k / b]+1$, it follows from Lemma 2.1 that (2.4) means that $p$ divides all the integers (2.3) since $j$ is arbitrary.

We next study the asymptotic behaviour of $d_{n}(\lambda ; a, b)$. First we consider the lower estimate. For an arbitrarily fixed integer $L>4 b^{2}$, let $I_{L}=(\alpha, \beta)$ be any connected component of the set $E_{L}(\lambda, k / b)$. Then any prime number $p$ with $p \equiv k^{\prime}(\bmod b)$ contained in the interval $J_{L}=(n / \beta \lambda, n / \alpha \lambda)$ must belong to the set $S_{n}(\lambda, k ; a, b)$ if $n>\max \left\{L, b(\beta \lambda)^{2}\right\}$. Hence we have

$$
\sum_{p \in S_{n}(\lambda, k ; a, b)} \log p \geq \pi\left(\frac{1}{\beta \lambda}, \frac{1}{\alpha \lambda} ; k^{\prime}, b ; n\right) \log \left(\frac{n}{\beta \lambda}\right),
$$

where $\pi\left(c, d ; k^{\prime}, b ; n\right)$ is the number of prime numbers $p \in(c n, d n)$ with $p \equiv k^{\prime}(\bmod b)$. Then, using the well-known prime number theorem for 
arithmetic progressions, we obtain

$$
\liminf _{n \rightarrow \infty} \frac{1}{n} \sum_{p \in S_{n}(\lambda, k ; a, b)} \log p \geq \frac{1}{\lambda \psi(b)}\left(\frac{1}{\alpha}-\frac{1}{\beta}\right)=\frac{1}{\lambda \psi(b)} \int_{I_{L}} \frac{d y}{y^{2}} .
$$

Therefore, since $L$ is arbitrary, it can be seen that

$$
\liminf _{n \rightarrow \infty} \frac{1}{n} \log d_{n}(\lambda ; a, b) \geq \frac{1}{\lambda \psi(b)} \sum_{\substack{1 \leq k<b \\(k, b)=1}} \int_{E(\lambda, k / b)} \frac{d y}{y^{2}},
$$

as required.

The desired upper estimate of $d_{n}(\lambda ; a, b)$ can be easily obtained by a similar argument. (Or one can make (2.5) an asymptotic equality just by replacing $d_{n}(\lambda ; a, b)$ by some subdivisor, which does exist since it is highly composite.) This completes the proof.

For brevity, we put

$$
e_{b}(\lambda)=\sum_{\substack{1 \leq k<b \\(k, b)=1}} \int_{E(\lambda, k / b)} \frac{d y}{y^{2}}
$$

If $\lambda=l \geq 2$ is an integer, then $e_{b}(l)$ can be written as a finite sum of values of the digamma function $\Gamma^{\prime}(z) / \Gamma(z)$ at rational points; hence, as a sum of values of elementary functions by Gauss' formula ([2], p.19). In particular, we have the following formulae for the cases in which $b=2,3$ and 4 :

$$
e_{2}(l)=-\log 2+\frac{\pi}{2}\{\chi(l+1)-\chi(l)\}
$$

where

$$
\begin{gathered}
\chi(l)=\sum_{j=1}^{l-1} \cot \left(\frac{j \pi}{2 l}\right) \\
e_{3}(l)=-\frac{3}{2} \log 3+\frac{\pi}{2}\left\{\chi_{2}(l+1)-\chi_{1}(l)\right\}
\end{gathered}
$$

where

$$
\begin{gathered}
\chi_{i}(l)=\sum_{j=1}^{l-1} \cot \left(\frac{j \pi}{3 l}\right)+\sum_{j=1}^{[l / 3]} \cot \left(\frac{j \pi}{l}\right)+\sum_{\substack{l<j<2 l \\
j \equiv i(\bmod 3)}} \cot \left(\frac{j \pi}{3 l}\right) \\
e_{4}(l)=-3 \log 2+\frac{\pi}{2}\left\{\widetilde{\chi}_{3}(l+1)-\widetilde{\chi}_{1}(l)\right\}
\end{gathered}
$$


where

$$
\tilde{\chi}_{i}(l)=\sum_{\substack{j=1 \\ j \neq 2(\bmod 4)}}^{l-1} \cot \left(\frac{j \pi}{4 l}\right)+\sum_{j=1}^{[l / 4]} \cot \left(\frac{j \pi}{l}\right)+\sum_{\substack{l \leq j<3 l \\ j \equiv i(\bmod 4)}} \cot \left(\frac{j \pi}{4 l}\right) .
$$

For example, for the proofs of Theorems 1.1, 1.2 and 1.3, we will use the exact values of $e_{2}(2), e_{3}(3)$ and $e_{4}(3)$ respectively.

3. Main theorem. To state our main theorem we need some definitions. Let $\lambda>1$ be a real parameter. For any integer $s \neq 0$, let $g_{\lambda}(s)$ be the unique solution of the quadratic equation

$$
(\lambda+1) x^{2}-s(2 \lambda+1) x+s \lambda=0
$$

in the unit interval $(0,1)$ and let $h_{\lambda}(s)$ be the second real solution of $(3.1)$; so, $h_{\lambda}(s)>s$ if $s>1$ and $h_{\lambda}(s)<s$ if $s \leq-1$. For any $x \in \mathbb{R}$, we define

$$
F_{\lambda}(x)=|x-1| \cdot\left|\left(2+\frac{1}{\lambda}\right) x-1\right|^{\lambda} .
$$

Finally, for any integer $b \geq 2$, we define

$$
\gamma_{\lambda}(b)=(\lambda+1) \frac{b}{\psi(b)} \sum_{\substack{1 \leq k<b \\(k, b)=1}} \frac{1}{k}-\left(\log b+\sum_{\substack{p \mid b \\ p \text { prime }}} \frac{\log p}{p-1}+\frac{e_{b}(\lambda)}{\psi(b)}\right) .
$$

Our main theorem can now be stated as follows:

TheOREM 3.1. Let $\delta=a / b \in(0,1)$ where $a, b$ are positive integers with $(a, b)=1$ and let $s \neq 1$ be an integer satisfying the condition (1.4). Suppose that there exists $\lambda>1$ satisfying

$$
\log F_{\lambda}\left(g_{\lambda}(s)\right)+\gamma_{\lambda}(b)<0 .
$$

Then, for any $\varepsilon>0$, there exists a positive integer $q(\varepsilon)$ such that

$$
\left|{ }_{2} F_{1}(1,1-\delta, 2-\delta ; 1 / s)-p / q\right| \geq q^{-\kappa-\varepsilon}
$$

for any integer $q \geq q(\varepsilon)$ and for all $p \in \mathbb{Z}$, where $\kappa$ is given by

$$
\kappa=1-\frac{\log F_{\lambda}\left(h_{\lambda}(s)\right)+\gamma_{\lambda}(b)}{\log F_{\lambda}\left(g_{\lambda}(s)\right)+\gamma_{\lambda}(b)} .
$$

Theorem 1.1 stated in Section 1 is now verified by taking $(\delta, s, \lambda)=$ $(1 / 2,5,2)$ in the above main theorem. Theorem 1.2 also follows by taking $(\delta, s, \lambda)=(1 / 3,-8,3)$ and $(2 / 3,-8,3)$. Similarly one has Theorem 1.3 by taking $(\delta, s, \lambda)=(1 / 4,9,3)$ and $(3 / 4,9,3)$. 
Proof. Let $D_{n}(a, b)$ be the least common multiple of $\{b-a, 2 b-a$, $\ldots, n b-a\}$. Then it is well known that

$$
\lim _{n \rightarrow \infty} \frac{1}{n} \log D_{n}(a, b)=\frac{b}{\psi(b)} \sum_{\substack{1 \leq k<b \\(k, b)=1}} \frac{1}{k} .
$$

(For the proof, see Alladi \& Robinson [1; Lemma 1].) For every $n \geq 1$, we put

$$
K_{n} \equiv K_{n}(\lambda ; a, b)=\frac{D_{n+m}(a, b)}{d_{n}(\lambda ; a, b)} b^{1-m} \prod_{\substack{p \mid b \\ p \text { prime }}} p^{C(n)-v(m-1, p)},
$$

where $C(n)=[2 \log (2 n)]$ and $m=[n / \lambda]$. Then it easily follows from (3.3) and Lemma 2.2 that

$$
\lim _{n \rightarrow \infty} \frac{1}{n} \log K_{n}=\gamma_{\lambda}(b) / \lambda .
$$

On the other hand, by the definition (1.2), we have

$$
\begin{aligned}
P_{n, m, \delta}(x) & =\frac{x^{\delta}}{n !} \sum_{j=0}^{n}\left(\begin{array}{c}
n \\
j
\end{array}\right)\left(x^{n-\delta}\right)^{(j)}\left((1-x)^{n+m}\right)^{(n-j)} \\
& =(-1)^{n} \sum_{j=0}^{n}\left(\begin{array}{c}
n-\delta \\
j
\end{array}\right)\left(\begin{array}{c}
n+m \\
n-j
\end{array}\right) x^{n-j}(1-x)^{j+m} \\
& =\sum_{j=0}^{n} A_{j, n} x^{n-j}(1-x)^{j+m}, \quad \text { say. }
\end{aligned}
$$

Note that each $A_{j, n}$ is a rational number, which is also dependent on $\lambda$ and $\delta$. Clearly we have from Lemma 2.2

$$
A_{j, n} \in \frac{d_{n}(\lambda ; a, b)}{b^{j} \prod_{p \mid b, p \text { prime }} p^{v(j, p)}} \mathbb{Z}
$$

for every $0 \leq j \leq n$. We then define

$$
\begin{aligned}
Q_{n, \lambda, \delta}(x) & =K_{n} P_{n, m, \delta}(1-x) \\
& =K_{n} \sum_{j=0}^{n} A_{j, n} x^{j+m}(1-x)^{n-j} \equiv \sum_{j=m}^{n+m} B_{j, n} x^{j},
\end{aligned}
$$

where

$$
\begin{array}{r}
B_{j, n}=K_{n}\left[A_{j-m, n}-\left(\begin{array}{c}
n+m-j+1 \\
1
\end{array}\right)\right. \\
A_{j-m-1, n}+\ldots \\
\left.\ldots+(-1)^{j-m}\left(\begin{array}{c}
n \\
j-m
\end{array}\right) A_{0, n}\right] .
\end{array}
$$


Therefore, from (3.5), we have

$$
B_{j, n} \in \frac{D_{n+m}(a, b)}{b^{j-1} \prod_{p \mid b, p \text { prime }} p^{v(j-1, p)-C(n)}} \mathbb{Z}
$$

for $m \leq j \leq n+m$, since $v(j-1, p) \geq v(m-1, p)+v(j-m, p)$.

We now consider the following integral:

$$
p_{n} \equiv p_{n}(\lambda, \delta ; s)=s(1-\delta) \int_{0}^{1} \frac{Q_{n, \lambda, \delta}(1-s)-Q_{n, \lambda, \delta}(1-y)}{s-y} \cdot \frac{d y}{y^{\delta}} .
$$

Then we have immediately

$$
\begin{aligned}
Q_{n, \lambda, \delta}(1-s) & { }_{2} F_{1}(1,1-\delta, 2-\delta ; 1 / s)-p_{n} \\
= & s(1-\delta) \int_{0}^{1} \frac{Q_{n, \lambda, \delta}(1-y)}{s-y} \cdot \frac{d y}{y^{\delta}}=\varepsilon_{n}(\lambda, \delta ; s) \equiv \varepsilon_{n}, \quad \text { say . }
\end{aligned}
$$

We first show that $p_{n} \in \mathbb{Z}$. For brevity, put $\widetilde{s}=1-s$; so, $\widetilde{s}$ is neither 0 nor 1. Then we have from (3.6)

$$
\begin{aligned}
p_{n} & =-s(1-\delta) \int_{0}^{1} \frac{Q_{n, \lambda, \delta}(\widetilde{s})-Q_{n, \lambda, \delta}(y)}{\widetilde{s}-y} \cdot \frac{d y}{(1-y)^{\delta}} \\
& =-s(1-\delta) \sum_{j=m}^{n+m} B_{j, n} \int_{0}^{1}\left(\widetilde{s}^{j-1}+\widetilde{s}^{j-2} y+\ldots+y^{j-1}\right) \frac{d y}{(1-y)^{\delta}} \\
& =-s(1-\delta) \sum_{j=m}^{n+m} B_{j, n} \sum_{l=1}^{j} \widetilde{s}^{j-l} \int_{0}^{1} y^{l-1}(1-y)^{-\delta} d y .
\end{aligned}
$$

Here we note that

$$
\begin{aligned}
\int_{0}^{1} y^{l-1}(1-y)^{-\delta} d y=\frac{\Gamma(l) \Gamma(1-\delta)}{\Gamma(l+1-\delta)} & =\frac{b^{l}(l-1) !}{(b-a) \ldots(l b-a)} \\
\in & \frac{b^{l}}{D_{l}(a, b)} \prod_{\substack{p \mid b \\
p \text { prime }}} p^{v(l-1, p)} \mathbb{Z} .
\end{aligned}
$$

To see this, it suffices to show that

$$
v(l, p ; a, b)-v(l-1, p) \leq L_{p}
$$

for each prime number $p$ with $(p, b)=1$, where $L_{p}$ is the exponent of $p$ in the resolution of $D_{l}(a, b)$ into its prime factors. Since the left-hand side of 
(3.9) is equal to

$$
\sum_{j=1}^{L_{p}}\left(\left[\frac{l}{p^{j}}+\frac{w_{j}}{b}\right]-\left[\frac{l-1}{p^{j}}\right]\right)
$$

from Lemma 2.1, it is sufficient to show that

$$
\left[\frac{l}{p^{j}}+\frac{w_{j}}{b}\right]-\left[\frac{l-1}{p^{j}}\right] \leq 1
$$

for each $j \geq 1$. Suppose, on the contrary, that the left-hand side of the above inequality is greater than 1 for some $l$ and some $j$. Let $r$ be the least non-negative residue of $l$ to modulus $p^{j}$. Then clearly

$$
\left[\frac{r}{p^{j}}+\frac{w_{j}}{b}\right]-\left[\frac{r-1}{p^{j}}\right] \geq 2 .
$$

Since $r=0$ does not satisfy the above inequality, we have $r \geq 1$; hence $\left[(r-1) / p^{j}\right]=0$, so $\left[r / p^{j}+w_{j} / b\right] \geq 2$. This is clearly a contradiction, since $r<p^{j}$ and $w_{j}<b$. Thus we have proved (3.8).

For any integer $b \geq 2$, we put

$$
M(b)=b \cdot \prod_{\substack{p \mid b \\ p \text { prime }}} p .
$$

Then it follows from (3.7) and (3.8) that

$$
B_{j, n} \widetilde{s}^{j-l} \frac{\Gamma(l) \Gamma(1-\delta)}{\Gamma(l+1-\delta)} \in b\left(\frac{\widetilde{s}}{M(b)}\right)^{j-l} \mathbb{Z}
$$

for every $1 \leq l \leq j$ and $m \leq j \leq n+m$, since

$$
v(j-1, p)-v(l-1, p) \leq j-l+\left[\frac{\log (2 n)}{\log p}\right] \leq j-l+C(n) .
$$

Thus we have $p_{n} \in \mathbb{Z}$ as required, since $\widetilde{s} \equiv 0(\bmod M(b))$ by the condition (1.4).

Similarly we have

$$
q_{n} \equiv q_{n}(\lambda, \delta ; s)=K_{n} P_{n, m, \delta}(s)=Q_{n, \lambda, \delta}(\widetilde{s}) \in \mathbb{Z},
$$

since $B_{j, n} \widetilde{s}^{j} \in b(\widetilde{s} / M(b))^{j} \mathbb{Z}$ for every $j$. Thus we obtain

$$
q_{n 2} F_{1}(1,1-\delta, 2-\delta ; 1 / s)-p_{n}=\varepsilon_{n}
$$

for some integers $p_{n}$ and $q_{n}$.

Note that the coefficient of $y^{j}$ of the polynomial $Q_{n, \lambda, \delta}(1-y)$ is

$$
(-1)^{j} K_{n}\left(\begin{array}{c}
n+m \\
j
\end{array}\right)\left(\begin{array}{c}
n+j-\delta \\
n
\end{array}\right)
$$

which is not an integer in general. 
In order to obtain an irrationality measure of ${ }_{2} F_{1}(1,1-\delta, 2-\delta ; 1 / s)$ from the approximation (3.10), we first study the asymptotic behaviour of the remainder terms $\left\{\varepsilon_{n}\right\}$. We have

$$
\begin{aligned}
\varepsilon_{n} & =s(1-\delta) K_{n} \int_{0}^{1} \frac{P_{n, m, \delta}(y)}{s-y} \cdot \frac{d y}{y^{\delta}} \\
& =s(1-\delta)(-1)^{n} K_{n} \int_{0}^{1} \frac{y^{n-\delta}(1-y)^{n+m}}{(s-y)^{n+1}} d y
\end{aligned}
$$

from an $n$-fold partial integration; hence from (3.4) we have

$$
\begin{aligned}
\lim _{n \rightarrow \infty} \frac{1}{n} \log \left|\varepsilon_{n}\right| & =\gamma_{\lambda}(b) / \lambda+\log \left(\max _{0 \leq y \leq 1} \frac{y(1-y)^{1+1 / \lambda}}{|s-y|}\right) \\
& \equiv-\tau / \lambda, \quad \text { say . }
\end{aligned}
$$

Then it can be seen that the maximum of the right-hand side of (3.11) is attained at $y=g_{\lambda}(s)$ and that from (3.2)

$$
\tau=-\gamma_{\lambda}(b)-\log F_{\lambda}\left(g_{\lambda}(s)\right)>0 .
$$

We next study the asymptotic behaviour of $\left\{q_{n}\right\}$. Let $C$ be the circle centered at $z=\varrho^{2} /\left(\varrho^{2}-1\right)$ with radius $\varrho /\left(\varrho^{2}-1\right)$, where

$$
\varrho=\frac{h_{\lambda}(s)}{h_{\lambda}(s)-s}>1 \text {. }
$$

Then it follows from Cauchy's integral formula that

$$
q_{n}=Q_{n, \lambda, \delta}(\widetilde{s})=K_{n} P_{n, m, \delta}(s)=\frac{K_{n}}{2 \pi i} \int_{C} \frac{z^{n-\delta}(1-s z)^{n+m}}{(z-1)^{n+1}} d z ;
$$

therefore

$$
\begin{aligned}
& \limsup _{n \rightarrow \infty} \frac{1}{n} \log \left|q_{n}\right| \\
& \quad \leq \gamma_{\lambda}(b) / \lambda+\log \left(\max _{z \in C}\left|\frac{z}{z-1}\right| \cdot|1-s z|^{1+1 / \lambda}\right) \equiv \sigma / \lambda, \quad \text { say . }
\end{aligned}
$$

It can be easily verified that the maximum of the right-hand side of (3.12) is attained at $z=\varrho /(\varrho-1)$ and that

$$
\sigma=\gamma_{\lambda}(b)+\log F_{\lambda}\left(h_{\lambda}(s)\right) .
$$

Thus, by Lemma 3.1 in [4], the number ${ }_{2} F_{1}(1,1-\delta, 2-\delta ; 1 / s)$ has an irrationality measure $1+\sigma / \tau$. This completes the proof of Theorem 3.1.

Acknowledgement. The author would like to thank the referee for suggesting many improvements and correcting some minor errors. 


\section{References}

[1] K. Alladi and M. L. Robinson, Legendre polynomials and irrationality, J. Reine Angew. Math. 318 (1980), 137-155.

[2] A. Erdélyi et al., Higher Transcendental Functions, Vol. 1, New York 1953.

[3] M. Hata, On the linear independence of the values of polylogarithmic functions, J. Math. Pures Appl. 69 (1990), 133-173.

[4] - Legendre type polynomials and irrationality measures, J. Reine Angew. Math. 407 (1990), 99-125.

[5] M. Huttner, Irrationalité de certaines intégrales hypergéométriques, J. Number Theory 26 (1987), 166-178.

[6] E. Reyssat, Mesures de transcendance pour les logarithmes de nombres rationnels, in: Progr. Math. 31, Birkhäuser, 1983, 235-245.

[7] G. Rhin, Approximants de Padé et mesures effectives d'irrationalité, in: Progr. Math. 71, Birkhäuser, 1987, 155-164.

INSTITUTE OF MATHEMATICS

YOSHIDA COLLEGE

KYOTO UNIVERSITY

KYOTO 606, JAPAN

Received on 10. 9. 1990

and in revised form on 13.5.1991 